\title{
A Survey on Different Techniques for Product Fake Review Detection and Product Rating
}

\author{
Adnan Telwala a,1, Ayush Pratap a , Ketan Gaikwad a, Tushar Chaudhari,Sukhada \\ Bhingarkar ${ }^{\text {a }}$ \\ ${ }^{\text {a }}$ School of Computer Engineering and Technology, MIT World Peace University, \\ Kothrud, Pune 411 038, Maharashtra - India
}

\begin{abstract}
Numerous online business sites empower the customers to create a product reviews along with feedback in the shape of ratings. This gives the organization work force a sign about their items' remaining on the lookout, while likewise empowering individual customer to frame an assessment and help buy an item. As of late, Sentiment Analysis (SA) has gotten quite possibly interesting due to the potential business advantages of text analysis. One of the most important problems in confronting SA is the manner by which to remove feelings in the assessment, as well as how to identify counterfeit good reviews and negative surveys derived from assessment surveys. Besides, the assessment surveys acquired from clients can divided into two categories: positive and negative, which can be utilized by a shopper to choose an item. In this survey, we have thoroughly discussed about fake review detection of products as well as product rating by different SA techniques. Further, we have discussed the research direction in fake review detection and product rating.
\end{abstract}

Keywords- Commercial benefits, Fake reviews, Opinion reviews, Opinion mining, Product rating, Sentiment analysis, Text analysis.

\section{Introduction}

As web-based business keeps on developing, the opposition among vendors for customers and deals has expanded essentially. A few traders decide to acquire an upper hand by utilizing misleading strategies to control online business stages, like the Amazon Marketplace. These beguiling strategies can incorporate controlling calculations, counterfeit item audits and evaluations just as snap ranches. Traders utilize these and different strategies to build their deals and gain piece of the overall industry. [1]

Ordinarily, a shipper will either boost its clients with blessings, money related remuneration or potentially guarantees to create and to compose an item survey or enroll the assistance of an outsider to creator an item audit. These reviews assist with driving buyer conduct; regardless of whether they are shopping through internet business stages or as our forefathers would have done it in retail outlets the nation over

\footnotetext{
${ }^{1}$ Adnan Telwala, School of Computer Engineering and Technology, MIT World Peace University, Email: adnantelwala31@gmail.com.
} 
Individuals depend on the audits and evaluations on Amazon when settling on buying choices [1]. The Washington Post nitty gritty in what way sellers make use of Facebook to help their Amazon assessments. The assessment questionnaire in December 2018 has discovered that $61 \%$ reviews for contraptions are fake, and in the meantime, overviews for supplements are 64\% phony. One more assessment in 2019 discovered that $82 \%$ of reviews read by the buyers are fake. [2] The fictitious product reviews are made and recorded either based on the dealer's item or on its rivals' items. On account of the contender, they may have a case identified with slander since the surveys will adversely misrepresent their item and its highlights. Then again, when the fake reviews are recorded on the trader's item, the rival may have a case identified with fake promoting and the reviews would be constructive misrepresent the dealer's own item and its highlights, accordingly arrogate deals from the contender. [2]

Existing methodologies for fake review detection and product rating are as follows:

Opinion Mining (OM) otherwise called Sentiment Analysis (SA) is the area of research that analyze individual's opinion, assessments, conclusions, sentiments, evaluations, and feelings towards substances like services people, issues, subjects, and their properties. There are various automated techniques for Sentiment Analysis. Machine learning techniques in addition with SA techniques are relied upon to own a significant beneficial outcome, particularly for fake review detection in product reviews, social networking sites, and different domains. There are diverse AI based strategies accessible like Support Vector Machine (SVM), Random Forest, and K-Nearest Neighbors (KNN) that are applied for the classification purposes. [3]

The remaining of the paper is organized as follows: Section II discusses about Fake Review Detection based on Sentimental Analysis. Section III throws a light on fake review detection features Section IV concludes the paper.

\section{Fake Review Detection based on Sentimental Analysis}

\subsection{Iterative Computer Framework $(\mathrm{ICF}++)$}

Iterative Computer Framework ( $\mathrm{ICF}++)$ [4] starts with a direct execution of a little arrangement of the product prerequisites and iteratively improves the propelling structures until the absolute system is done and fit to be sent.

$\mathrm{ICF}++$ measures the trustworthiness estimation of a review by using the content mining and assessment mining methods. The name of an item, the name of an observer, the content of a survey, the amount of focus, and minsup as a boundary are all used in the ICF++ methodology. This technique adds the counter to nothing, genuineness value, and dependability worth to one after taking certain attributes. From that point onward, the following cycles are Parts of Speech (POS) labeling, production of transaction file, frequent pattern (FP) growth, generation of polarity, agreement value calculation.

Iteration is the next step. In each iteration, the system calculates the fairness, trustworthiness, and reliability values, as well as updating the agreement attribute with the updated integrity, reliability, and increment counter. A major advantage of the $\mathrm{ICF}++$ is that the accuracy is enhanced. The disadvantage of this approach is that 
certain processes must be streamlined in order for it to detect a fake review in a reasonable period of time.

Further steps used in ICF++ are as follows:

- $\quad$ Parts Of Speech (POS) tagging: The Stanford Part-Of-Speech (POS) tagger from stanfordcorenlp-3.5.1 [5] is used for POS tagging. POS Tagger is a product that receives messages and selects the appropriate linguistic structure for each token. For English taggers, this connection transmitted a tag as shortenings, and the truncation's Penn Treebank standard is used. A Java-based work territory framework is created for this communication. The setup of the tagger model was the first step in this process. This connection's commitment is a sentence taken from the analysis and development of a POS tag for each component that is inserted into the database.

- Creation of transaction file: All tokens that are stored in the database from objects are included in the transaction file analysis. It has the tag value of noun (NN), noun pronoun (NNP), noun pronoun singular (NNPS), or noun singular (NNS). Each object review is represented by a noun in the transaction file. This transaction file is used to calculate the FP Growth contribution.

- Frequent Pattern (FP) Growth: This communication involves understanding the highlights that have received the most reviews. The highlights of this investigation are the properties or characteristics of an item. For example, the highlights of a camera may be the battery, memory card, and so on. This data is available on Amazon, but datasets associated with it are not, and so to get data about an item's component, this technique has used FP-development measurement from affiliation rule mining tool.

\subsection{Graph based Model for Fake Review Detection}

This section focuses on graph based fake review detection in context of online product reviews. This model replaces store nodes with product nodes and introduces new scoring criteria to capture the intricate relationships between all kinds of nodes. Moreover, this model exploits more features relating to each kind of nodes to govern the spam city of reviews, reviewers, and products, which can achieve a remarkable precision improvement.

Here are some node features in product review graph. These features are related with reviewers, reviews and products [6].

\subsection{Reviewer-related features:}

Reviewer's Review Content Similarity (RRCS): Fake reviewers are likely to copy (or slightly modify) their previous reviews across similar products to save time and energy. RRCS of " $r$ " is the average value of the entire cosine similarities between each review text pairs written by reviewer.

$$
R R C S(r)=\operatorname{avg}_{v_{i}, v_{j}} \in V_{r, i}<j^{\operatorname{cosine}\left(v_{i}, v_{j}\right)}
$$


Average Rating Score (ARS): If a reviewer's average rating score is too low, then that reviewer intends to be a spammer.

$$
A R S(r)=\left(5-\operatorname{avg}_{v \in V_{r}} \Psi_{v}\right) / 4
$$

Where $\Psi v$ is the rating score of review $v$ in a 5-star rating system.

Reviewer Active Duration (RAD): Opinion spammers are normally newcomers to a website. Let $L(r)$ and $F(r)$ be the last and first review date of reviewer $r, T$ be a userspecified time threshold, then:

$R A D(r)=\left\{\begin{array}{c}1-\frac{L(r)-F(r)}{T}, L(r)-F(r) \leq T \\ 0, L(r)-F(r)>T\end{array}\right.$

Ratio of First Reviews (RFR)

Review spammers intend to post reviews early to influence the sentiment of a product. This function is described as the ratio of the number of reviews in the first $\mathrm{k} \%$ of a product's reviews to the total number of reviews written by the reviewer $r$ :

$$
R F R(r)=\frac{\mid\left\{v \mid v \in V_{r}, v \text { is in the first } k \% \text { reviews }\right\} \mid}{\left|V_{r}\right|}
$$

Review Date Entropy (RDE): Genuine reviewers usually post reviews spontaneously, while review spammers often submit a significant number of reviews in a limited period of time (e.g., one day) to gain the maximum profits. This model feature by the entropy of review dates of that reviewer. Clearly, spammers have lower review date entropies than those of genuine reviewers.

$$
R D E(r)=1+\sum_{d} \frac{\left|V_{r}^{d}\right|}{\left|V_{r}\right|} \log _{\left|D_{r}\right|}\left(\frac{\left|V_{r}^{d}\right|}{\left|V_{r}\right|}\right)
$$

\subsection{Review-related features}

1. Review Content Similarity (RCS): For a single review $\mathrm{v}$ about product $\mathrm{p}$, let $\mathrm{V}_{p}$ be p's review set, then RCS is defined as the maximum cosine similarity of review $\mathrm{v}$ and its earlier review $\mathrm{v}^{\prime}$ in $\mathrm{V}_{p}$ :

$$
R C S(v)=\max _{v^{\prime} \in V_{p}, \operatorname{date}\left(v^{\prime}\right)<\operatorname{date}(v)} \operatorname{cosine}\left(v, v^{\prime}\right)
$$

2. Rating Deviation (RD):

Review spammers want to advertise or degrade their products, because their reviews for the same product which differ significantly from those of other reviewers. This model features as:

$$
R D(v)=\frac{\left|\Psi_{v}-a v g_{v^{\prime} \in V_{p}} \Psi_{v^{\prime}}\right|}{4}
$$

\subsection{Product-related features}

1. Average Rating (AR): The average rating score of product $p$ reflects the quality of that product. Average rating is defined as: 
$A R(p)=\frac{5-a v g_{v \in V} \Psi_{v}}{4}$

Total Number of Reviews (TNR)- Good quality products deserve more reviews.

Let $P$ be the product set, then

$$
\operatorname{TNR}(p)=1-\frac{\left|V_{p}\right|}{\max _{p^{\prime} \in \rho}\left|V_{p}\right|}
$$

\subsection{Iterative Computation with Elimination (ICE)}

The computation framework can also be applied to product review graph model. However, because the number of products are considerably larger than that of stores, the computation will take more time than with stores. Therefore, this framework proposes a refined algorithm ICE to compute the node scores efficiently, as shown below. An advantage of this framework is that it is a novel algorithm to efficiently compute the reputation scores of each kind of nodes in the graph. However, Individual spam features (ISF) had a weak output. Linguistic features (LF) did not do well.

Algorithm: ICE (Iterative Computation with Elimination)

Input: reviewer set $\mathrm{R}$, review set $\mathrm{V}$, product set $\mathrm{P}$, time window $\Delta \mathrm{t}$, number of reviewers to be returned $\mathrm{N}$, elimination rate $\rho$

Output: Fake reviewer set $\mathrm{F},|\mathrm{F}|=\mathrm{N}$, in ascending order of trustiness of reviewers

Step 1: Set all trustiness of reviewers to 1, set all reliability of products to 1, mark all reviewers in $\mathrm{R}$ as not eliminated;

Step 2: Repeat until the top $\mathrm{N}$ fake reviewer set $\mathrm{F}$ does not change or the maximum number of iterations reached

Step 2.1: Compute the honesty of reviews by non-eliminated reviewers according to

Step 2.2: Compute the trustiness of non- eliminated reviewers according to

Step 2.3: Compute the reliability of products in $\mathrm{P}$ according to

Step 2.4: Eliminate top $\rho$ percent of non- eliminated reviewers based on their trustiness scores in descending order.

Step 3: Return F [6]

\section{Fake Review Detection Features [7]}

The features used for fake review detection are as follows: - 
3.1. Duplicate reviews and rating: Repeated reviews of a product, comments on a product and rating consistently for a similar product or service by same ID.

3.2. Username consisting numbers: A true consumer or customer must show his or her actual name, which cannot be made up entirely of numbers. Since the genuine buyer would have a name and an address in alphanumeric notation, the only number means spammer.

3.3. Only star rating: Leaving a star rating would not guarantee a positive score and customers will want to suggest more before leaving a star rating. As a result, only star ratings are deemed fraudulent.

3.4. No category Details: Real purchaser will write the highlights and specifications of product in his reviews and then rate his experience of using the item. As a result, it is marked fake if a person posts a review without specifying the product classification or spotlight.

3.5. Review Sentiment and rating: These reviews are deemed fraudulent whether they have the highest rating but poor sentiment, or opposite, - for example if a user posts a positive rating but his or her review responses do not fit the rating.

3.6. Size of review: An authentic purchaser will leave a comment stating what he liked and did not like about the product or service when addressing the functionality of the product or service. As a result, the review must adhere to a strict size of review.

\section{Discussion}

Just single factor may not give exact recognizable proof of fake reviews. Consequently, value of all variables is considered in the review measure. This paper presents a review of some most prominent fake review detection method for product rating. Figure 1 below summarizes the capabilities of existing methods along with the limitations that indicate further improvement and future work.

\section{Results}

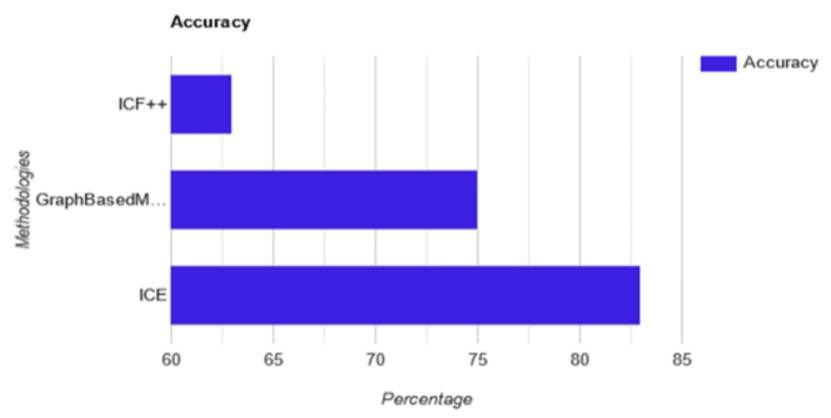

Figure 1. Existing methods along with the limitations that indicate further improvement and future work. 


\section{Conclusion}

It is difficult to go through and review manually to purchase a new product in today's world, where each product has thousands of reviews available. Many of these reviews are spam or fraudulent, or they are focused on consumers' sentimental loyalty to a particular company or competitor. Therefore, there is need for detection of fake reviews. In this survey paper, several proposed SA methods are discussed like ICF++, ICE, Graph based model. Not all papers summarized in this survey have claimed accuracy rate. For $\mathrm{ICF}++$ method, claimed accuracy rate is $63 \%$ and the limitation is that the process should be optimized for it to work in short amount of time and for ICE algorithm, the complexity of the system increases very much and accuracy is low. The graph-based model is having $75 \%$ accuracy but it significantly slows as the size of that data in use grows. However, accuracy can be increased by using machine-learning techniques like SVM, Random Forest etc. to $85 \%$. For future work, we will apply machine learning techniques and sentimental analysis, which will increase accuracy furthermore.

\section{References}

[1] Risk management in software. (2020, October 02). Retrieved from https://www.castsoftware.com/research-labs/risk-management-in-software development-andsoftware-engineering- projects.

[2] E-commerce manipulation the fake review. (2020, January 2) Retrieved from https://kjk.com/2020/01/25/e-commerce-manipulation-the-fake-review- problem/

[3] Fake-reviews detection. (2021, February 17). Retrieved from https://www.cs.uic.edu/ liub/FBS/fake- reviews.html

[4] Arif Djunaidy and Eka Dyar Wahyuni. Fake Review Detection from a Product Review Using Modified Method ofIterative Computation Framework MATEC Web of Conferences 58, 03003 (2016)

[5] POS tagger (2021, March 09). Retrieved from https://nlp.stanford.edu/software/tagger.shtml

[6] Dawei SONG, Tingting HOU, Zhun LI, Zhuo WANG. Spotting Fake Reviewers using Product Review Graph Journal of Computational Information Systems 11: 16 (2015) 5759-5767 Available at http://www.Jofcis.com

[7] Ms. Rajshri P. Kashti1, Dr. Prakash S. Prasad Enhancing NLP Techniques for Fake Review Detection International Journal of Pure and Applied Mathematics Volume 119 No. 12 2018, 13203-13209 